\title{
Recognition and Importance of New Definitions of Peripancreatic Fluid Collections in Managing Patients with Acute Pancreatitis
}

\author{
Akram Ahmed Waleed Gibreel Michael G. Sarr \\ Department of Surgery, Mayo Clinic, Rochester, Minn., USA
}

\section{Key Words}

Acute pancreatitis · Interstitial edematous pancreatitis .

Necrotizing pancreatitis - Acute peripancreatic fluid collection - Pancreatic pseudocyst - Acute necrotic collection - Walled-off necrosis · Disconnected duct syndrome

\begin{abstract}
Our understanding of the etiopathogenesis of acute pancreatitis has matured tremendously in the last 3 decades. Advanced cross-sectional imaging with 3-dimensional techniques along with use of intravenous contrast to image the presence or absence of organ tissue perfusion has allowed early recognition of necrotizing pancreatitis. With this knowledge, the old terms to describe what used to be called 'peripancreatic fluid collections' we now recognize are no longer accurate nor appropriate. The 2013 revised Atlanta Classification has introduced a new, accurate, objective classification of acute pancreatitis and terminology for the natural history of all forms of acute pancreatitis that is easy to use and will help in both the description of the disease and its appropriate treatment. This review will describe these pancreatic and peri-pancreatic collections with added insight into their natural history.

(c) 2016 S. Karger AG, Basel
\end{abstract}

(c) 2016 S. Karger AG, Basel

$0253-4886 / 16 / 0334-0259 \$ 39.50 / 0$

\section{Introduction}

The management of pancreatic and peri-pancreatic collections that complicate acute pancreatitis, chronic pancreatitis, pancreatic surgery and pancreatic trauma have been the subject of considerable controversy in the literature. Reasons for this controversy include the following: different techniques of management, different etiologies, different parochial views/beliefs in their natural history, etc. But one of the major reasons for apparent differences in treatment and primarily outcomes are secondary to miscommunication between groups and marked differences in the understanding of much of the terminology used in the past and currently to describe these collections. To date, there has been no universally accepted classification of these collections. All too often, these collections, despite being combinations of both liquid and solid components, are labeled under the non-specific global terms 'pancreatic pseudocyst' or 'pancreatic (or peri-pancreatic) fluid collections'. Unfortunately, these terms, when used indiscriminately in these clinical scenarios, are potentially confusing and misleading to surgeons. The term 'pancreatic pseudo-

Drs. A. Ahmed and W. Gibreel are co-first authors.

\section{KARGER}

E-Mail karger@karger.com

www.karger.com/dsu
Dr. Michael G. Sarr, MD

Department of Surgery

Mayo Clinic, 200 First Street, SW

Rochester, MN 55905 (USA)

E-Mail sarr.michael@ mayo.edu 
cyst' to most pancreatologists represents a very specific term, implies a different pathogenesis than the other collections and is managed differently than the other pancreatic and peri-pancreatic collections. Similarly, the terms 'pancreatic pseudocyst' or 'pancreatic fluid collection' implies a 'fluid' collection without any solid component, and when used in patients with severe acute pancreatitis, especially if it is necrotizing pancreatitis, is usually not accurate and overlooks the associated solid components that become very important clinically in subsequent management and prognostication. This uncertainty about the nature of these 'pseudo' pseudocysts and 'fluid collections' has been present for decades in the literature and has led to tremendous confusion in the appropriate management and the understanding of the natural history of these collections. The aim of this review is an attempt to provide an objective classification of peri-pancreatic and pancreatic collections as proposed in part by the 2013 revision of the 1992 Atlanta Classification $[1,2]$.

Why the emphasis on this terminology and this topic? The history of 'pancreatic pseudocysts' goes back many years to the era before CT. The diagnosis was made by clinical examination (a palpable mass) or by an upper gastrointestinal contrast examination showing displacement of the stomach or the duodenum by a non-visualized, extraluminal mass. Introduction of cross-sectional imaging, first transcutaneous ultrasonography (U/S), then CT and now MRI and endoscopic U/S allow the imaging of organs in spaces outside the gut lumen, thereby providing the ability to 'see' fluid collections in and around the pancreas. Use of intravenous contrast during the CT also allowed visualization of vascular perfusion of the pancreatic parenchyma by use of intravenous contrast agents, which for once allowed the recognition of actual pancreatic parenchymal necrosis non-invasively early in the course of the disease (the first week) before the need for any operative intervention. This recognition of non-perfusion of the parenchyma also changed our understanding of some elements of the pathogenesis of the disease.

The fact that non-perfusion and hence necrosis of the pancreatic parenchyma can be recognized in as early as the first $12 \mathrm{~h}$ using contrast-enhanced CT (CECT) led to the introduction of a new term - 'necrotizing pancreatitis', which implied an ischemic phenomenon and not the now outdated misnomer of 'hemorrhagic pancreatitis'. Unfortunately, many physicians assumed that the 'necrosis' only involved the pancreatic parenchyma, and any other fluid or partially fluid-filled collection was a 'pancreatic pseudocyst' or (worse yet!) a 'phlegmon'. This confusion arose, because 'perfusion' of the peri-pancreat- ic retroperitoneal fat is not visible normally, and the peripancreatic retroperitoneal fat does not 'enhance' with intravenous contrast; thus the lack of perfusion (and thereby the viability) of the peri-pancreatic fat cannot be evaluated directly. Although all surgeons who operated on severe acute pancreatitis or 'pancreatic abscess' knew that severe pancreatitis usually had associated 'fat necrosis', the 'necrosis' of this fat is not an objective diagnosis able to be made on CECT, but can be assumed with insight into the natural history of the disease combined with the clinical presentation. In addition, the appreciation of peri-pancreatic necrosis in the absence of peri-pancreatic parenchymal necrosis was not really accepted until the paper by Sakorafas et al. [3] was published in 1999 and more recently reinforced by others [4]. Since then, our understanding of acute necrotizing pancreatitis has eventuated in the revision of the original 1992 Atlanta Classification, which did not recognize/acknowledge this concept of peri-pancreatic necrosis $[1,5]$ and tried to introduce new terms that further confused the issue [6].

Our current understanding of this classification of acute pancreatitis is the following (table 1). The process is either an edematous pancreatopathy without necrosis or even much active inflammation (hence the term 'interstitial edematous pancreatitis'), or it is a 'necrotizing pancreatitis' with the necrosis affecting: (1) some of the pancreatic parenchyma and the peri-pancreatic tissues (about $80 \%$ of cases), (2) only the peri-pancreatic tissues with no evident pancreatic parenchymal necrosis (about 20\%) or (3) rarely, just the pancreatic parenchyma alone $(<5 \%)$. With this current understanding, most CECTs combined with the clinical scenario can be interpreted according to this new classification of acute pancreatitis based on insight into appearances on CECT [4]. Despite no obvious contrast enhancement or lack thereof in these retroperitoneal tissues, the appearance of what in the past was termed a phlegmon is now recognized as peri-pancreatic necrosis leading to the new term 'acute necrotic collection (ANC)', which brings in a different etiology, treatment and prognosis into the clinical scenario, which differs markedly from that of a true pancreatic 'pseudocyst'. With this in mind, much of the past literature on 'pancreatic pseudocysts' in the setting of acute pancreatitis needs to be reinterpreted, and the terms 'phlegmon', 'pancreatic abscess' and 'hemorrhagic pancreatitis' should no longer be used.

The suspicion of the different pancreatic and peri-pancreatic collections originating as local complications of acute pancreatitis can be expected usually based on the clinical presentation, but abdominal imaging has become the cornerstone of diagnosis. CECT remains the diagnos- 
tic modality of choice at most centers, although MRI is appropriate in centers with expertise with this technique and may in the future replace CECT. For CECT, the pancreas is best visualized during the portal venous phase, which is about 60-75 s after intravenous contrast injection. The normal pancreas shows complete enhancement with a density between 100 and 150 Hounsfield units. Pancreatic necrosis appears as areas that lack enhancement with the intravenous contrast material. Other diagnostic modalities, U/S and MRI, can serve as adjuncts to CECT and will often visualize the solid and liquid components better than CECT; unfortunately, U/S is all too often obscured by overlying gas, while MRI is less readily available, more expensive and requires expertise in its interpretation both by the radiologist and the surgeon. For these reasons, CECT will be the focus in this review, highlighting the characteristic radiologic features of the different pancreatic and peri-pancreatic collections. Based on the new understanding and classification of acute pancreatitis, combined with the correct interpretation of those radiologic findings, 4 different entities of pancreatic and peri-pancreatic collections with specific descriptive terms can be characterized.

\section{Terminology of Pancreatic and Peri-Pancreatic Collections}

\section{Acute Peri-Pancreatic Fluid Collections}

Etiopathogenesis

Acute peri-pancreatic fluid collections (APFCs) can complicate acute interstitial edematous pancreatitis and can be evident in up to $30-50 \%$ cases of acute interstitial edematous pancreatitis [1]. These collections develop early after the onset of pancreatitis due to extravasation of fluid secondary to an increased capillary permeability associated with the pancreatic injury. Very little direct acinar cell necrosis occurs via the pathogenesis of interstitial acute pancreatitis. This pathogenesis is basically a different process than acute necrotizing pancreatitis. These non-localized fluid collections (APFCs) lack an encapsulated wall and usually lie within the confines of tissue planes. Interestingly, the fluid does not contain an increased activity of pancreatic enzymes. The pancreas usually shows features consistent with interstitial edematous pancreatitis, and the pancreatic parenchyma enhances completely without evidence of necrosis; there may, however, be a bit more patchy enhancement due to the edema in the interstitium. A solid component is absent from an APFC. When solid components are present, the diagnosis of APFC should be questioned.

Importance of Terminology of Collections in Necrotizing Pancreatitis
Table 1. Terminology of acute pancreatitis - revised Atlanta classification [5]

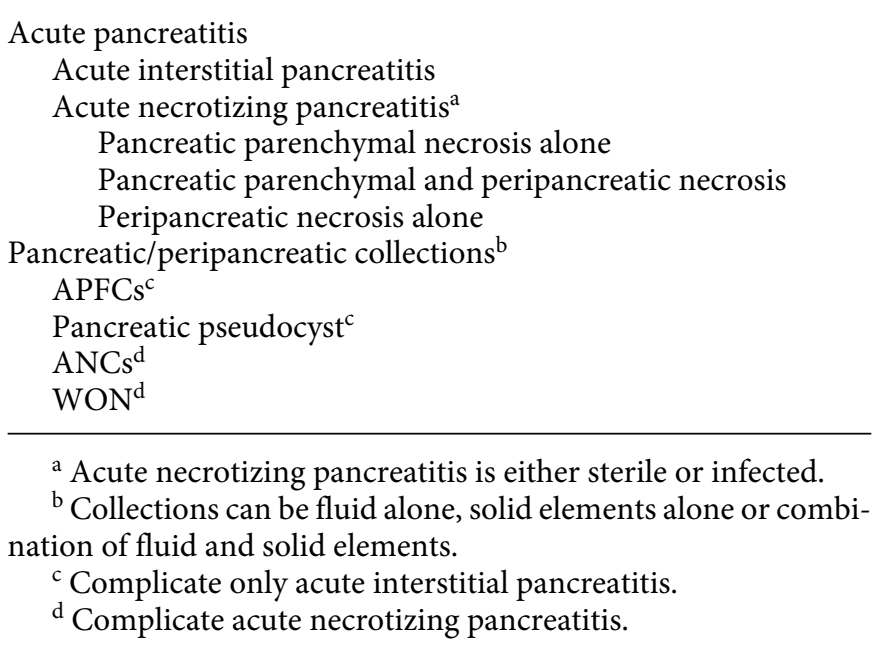

\section{Imaging Characteristics}

APFCs appear as ill-defined fluid collections developing between tissue planes (table 2). These collections lack a 'wall' or 'encapsulation' and appear to 'dissect' along defined transitions from the pancreatic parenchyma and the peri-pancreatic tissues (fig. 1). There is usually also an element of pancreatic parenchymal 'edema' as well.

\section{Natural History}

The natural history of APFCs usually follows a gradual course of spontaneous resolution. Among patients who develop an APFC, about 70\% show complete resolution within 2 weeks from the onset of pancreatitis [7]. If the collection persists beyond 4 weeks, it is likely to become encapsulated and at this stage is called a pancreatic pseudocyst. Of note, the progression to pancreatic pseudocyst is a rare occurrence (about $7 \%$ ) in patients who initially develop APFCs. There are no known factors that predict progression of an APFC to a pancreatic pseudocyst able to be determined by our classic methods of imaging (CT, MRI, U/S).

\section{Peri-Pancreatic Pseudocysts}

Etiopathogenesis

When acute peri-pancreatic fluid collections persist beyond 4 weeks, they have typically developed a recognizable wall of inflammatory, reactive granulation tissue. This 'pseudocyst' lacks an epithelial lining, which differentiates it from a true pancreatic cyst. The majority of classic pancreatic pseudocysts complicates acute interstitial edematous pancreatitis and develops as a result of 
Fig. 1. APFC and pseudocyst. APFC early in course of disease. See rim of fluid outside the pancreatic parenchyma following the peri-pancreatic planes and enhancement of the pancreatic parenchyma.

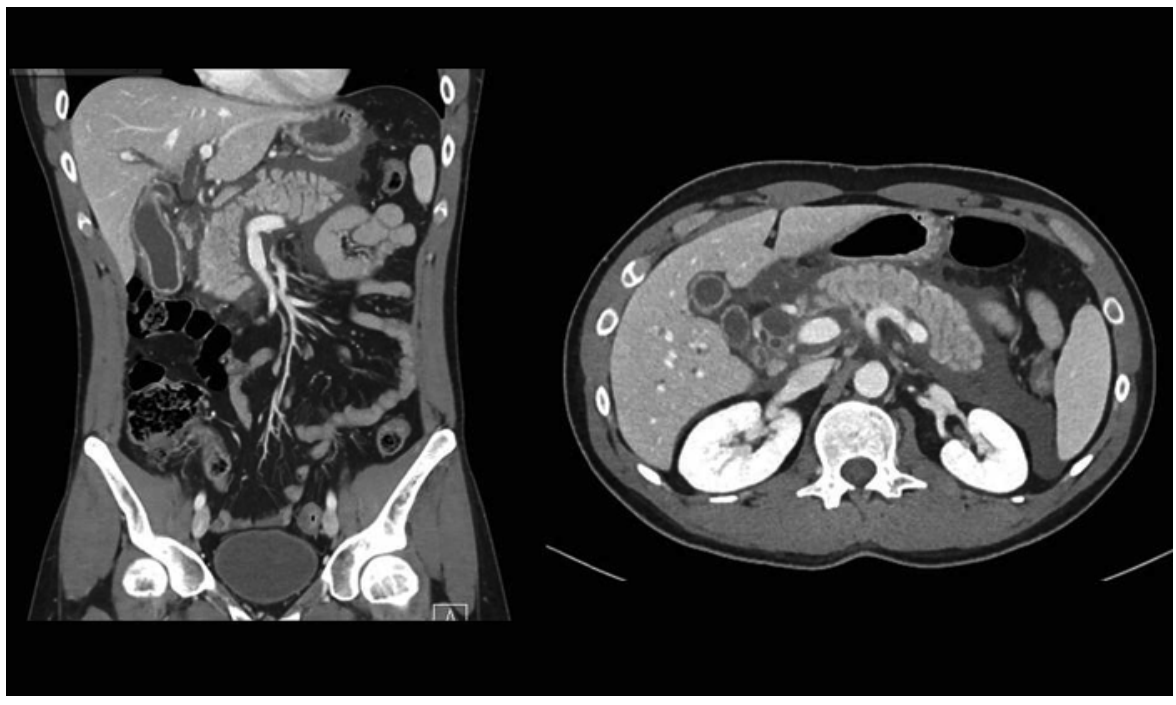

Table 2. Shows the time frame of development relative to the onset of pancreatitis, CECT features and natural history of peri-pancreatic fluid collections. The time of onset is defined as the time of onset of abdominal pain

\begin{tabular}{|c|c|c|c|}
\hline & $\begin{array}{l}\text { Time since } \\
\text { onset, weeks }\end{array}$ & CECT features & Natural history \\
\hline APFC & $<4$ & $\begin{array}{l}\text { Homogeneous ill-defined fluid density } \\
\text { Lacks encapsulated wall } \\
\text { No evidence of pancreatic necrosis (pancreas is completely } \\
\text { enhancing) }\end{array}$ & $\begin{array}{l}70 \% \text { resolve within } 2 \text { weeks } \\
\text { Progresses to pseudocyst in } 7 \%\end{array}$ \\
\hline $\begin{array}{l}\text { Pancreatic } \\
\text { pseudocyst }\end{array}$ & $>4$ & $\begin{array}{l}\text { Homogenous rounded fluid density } \\
\text { Encapsulated wall } \\
\text { Typically peri-pancreatic } \\
\text { Complete enhancement of the pancreas with no evidence of } \\
\text { necrosis (with } 2 \text { exceptions) }\end{array}$ & $\begin{array}{l}\text { Usually resolves } \\
\text { Resolution rate is lower if } \\
\text { complicates chronic pancreatitis }\end{array}$ \\
\hline ANC & $<4$ & $\begin{array}{l}\text { Occurs only in the setting of necrotizing } \\
\text { Could be intrapancreatic/peri-pancreatic } \\
\text { Fluid collection is heterogenous (fluid and non-fluid densities) } \\
\text { It lacks a well-defined wall }\end{array}$ & Evolves into WON after 4 weeks \\
\hline WON & $>4$ & $\begin{array}{l}\text { Heterogenous fluid collection (liquid and non-liquid densities) } \\
\text { Completely encapsulated with a well-defined wall } \\
\text { Could be intra- or extrapancreatic }\end{array}$ & $\begin{array}{l}\text { About } 50 \% \text { will become } \\
\text { secondarily infected }\end{array}$ \\
\hline
\end{tabular}

some type of disruption of the main pancreatic duct or one of its intrapancreatic branches. Although this process during acute pancreatitis obviously involves a focal element of pancreatic parenchymal and/or ductal injury, there is not enough parenchymal injury to be recognized on cross-sectional imaging. Also, these pancreatic pseudocysts complicating acute pancreatitis are located anatomically outside the confines of the pancreatic parenchyma (i.e., peri-pancreatic).
A pancreatic pseudocyst, as defined here, can rarely complicate necrotizing pancreatitis in 2 specific situations. First, when necrotizing pancreatitis involves necrosis of the pancreatic neck that leaves the distal pancreatic remnant viable but 'disconnected' from the proximal necrotic pancreas [8], and after resolution of the proximal necrosis, the pancreatic remnant can continue to secrete pancreatic juices leading to a clear fluid collection into a pancreatic 'pseudocyst'; this clinical scenario has been 
termed the disconnected duct syndrome. Second, a similar situation can occur in a cavity in the pancreatic or peripancreatic region after an operative necrosectomy where all the necrosis has been removed mechanically (necrosectomy) and a ductal disruption persists secreting pancreatic juice into this post-necrosectomy cavity [9]; this form of pancreatic pseudocyst should probably be referred to as a 'post-necrosectomy pancreatic pseudocyst', which in itself implies a different pathogenesis.

Because the inflammatory wall of a pseudocyst is actually encapsulating pancreatic exocrine secretions, analysis of the aspirated cavity fluid reveals a very high amylase activity and lipase activity, but most of the other protease enzymes are less stable, and thus amylase activity seems to be the best marker for a pseudocyst.

\section{Imaging Characteristics}

Pancreatic pseudocysts complicating acute pancreatitis appear as a well-encapsulated, homogenous fluid density on CECT located outside the confines of the pancreas and specifically with minimal or no intraluminal necrosis or solid matter (fig. 2). The pancreatic parenchyma has a normal contrast enhancement. If the 'pseudocyst' cavity contains debris, solid material or necrosis, the term 'pseudocyst' should not be used [9]. U/S or MRI may be necessary to exclude the presence of solid components within the pseudocyst cavity. This concept is quite important in differentiating pancreatic pseudocysts from areas of ANCs and from areas of walled-off necrosis (WON). As will be seen below, these latter 2 entities are complications of acute necrotizing pancreatitis: have a different pathogenesis (substantial pancreatic parenchymal or peri-pancreatic tissue necrosis) and portend a greater morbidity and often different treatments. Another characteristic of pseudocysts are that they are typically round without lobulations; in contrast, the necrotic collections complicating necrotizing pancreatitis are often lobulated, less round and multiple.

\section{Natural History}

Peri-pancreatic pseudocysts occur in 3\% of patients with acute interstitial edematous pancreatitis and in about 7\% of cases when APFCs exist initially [7]. Initial studies in the 1970s by Bradley et al. [10] based on U/S reported a high complication rate and low resolution rate when a conservative, non-operative approach to then management of pancreatic 'pseudocysts' was followed even for asymptomatic 'pseudocysts'. These findings led to the adoption of an aggressive operative management over conservative management, but in retrospect, the ma-

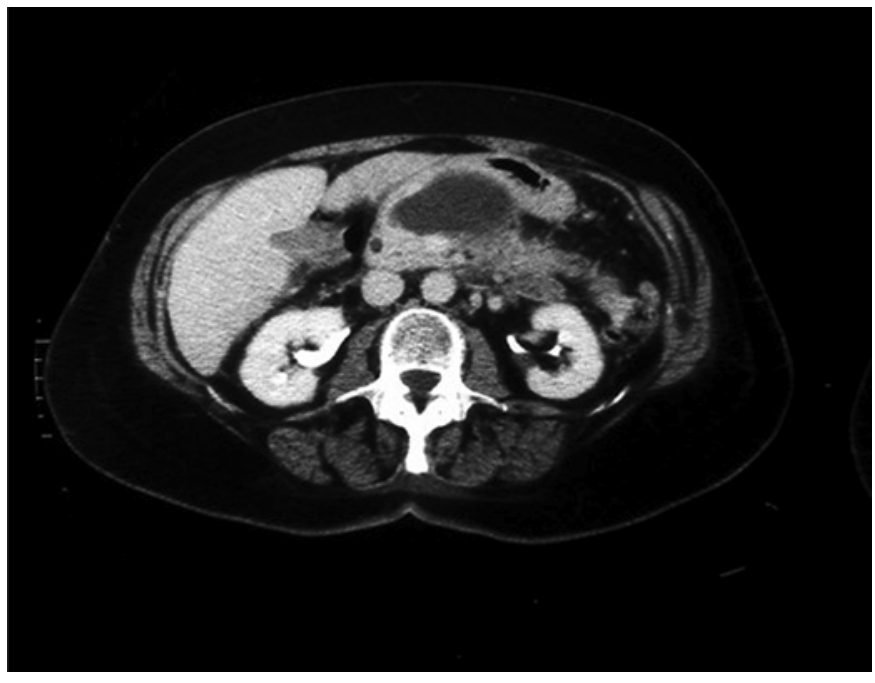

Fig. 2. Pancreatic pseudocyst. Note the fluid-filled extrapancreatic collection in this case outside the head of the pancreas.

jority of patients in this series that stimulated this suggestion of obligate operative intervention were alcoholics diagnosed by U/S, and most of who developed complications probably had necrotizing pancreatitis. Thus, many of these 'pseudocysts' were actually areas of ANC or WON. More recent studies by Vitas and Sarr [11] and Yeo et al. [12], however, revealed that most true pancreatic pseudocysts resolve without serious complications when managed non-operatively. Active intervention (endoscopic, percutaneous or open) is reserved for patients with symptoms or with an obviously enlarging pseudocyst. It should be emphasized that conservative management results in a greater resolution rate with pseudocysts originating in the setting of acute interstitial edematous pancreatitis versus pseudocysts arising in the setting of chronic pancreatitis; these latter pseudocysts are often intraparenchymal and involve a different etiopathogenesis.

\section{Acute Necrotic Collections}

Etiopathogenesis

ANCs result from acute necrotizing pancreatitis. The necrotizing process can affect the pancreatic parenchyma only, peri-pancreatic tissues only or most commonly both the pancreatic parenchyma and the peri-pancreatic tissues. The neck and proximal body of the pancreatic parenchyma is the anatomic region of the pancreas affected most commonly, while the left aspect of the peri-pancreatic retroperitoneal fat extending down the left gutter and often out along the base of the small bowel mesentery is the peri-pancreatic tissue involved most commonly. 
The normal pancreatic microcirculation is disrupted early during the acute necrotizing pancreatitis, and the affected portions of the pancreas, therefore, do not show contrast enhancement during CECT [13]. Whether the disruption of the microcirculation is a result of the acinar cell death or is itself part of the pathogenesis of the acute pancreatitis is unknown. The retroperitoneal 'fat' necrosis is a result of extravasation of pancreatic enzymes combined with the inflammatory mediators - cytokines, leukokines, etc. It should be emphasized that in the presence of pancreatic/peri-pancreatic necrosis, ANCs may initially lack a recognizable liquid component, because the full inflammatory response to the acute necrosis is not well established yet. Serial imaging will later reveal the presence of both solid and liquid components. When a solid component is identified, it is appropriate to use the term ANC rather than APFC to describe these collections, because APFCs by definition lack a solid component.

\section{Imaging Characteristics}

ANC occur only in the setting of acute necrotizing pancreatitis. After the first several days of the disease, these collections appear as non-homogeneous areas both within and outside the pancreatic parenchyma of both solid and liquid components representative of areas of necrosis, which stimulated an inflammatory influx of cytokines, inflammatory cells and inflammatory exudate (fig. 3, table 2). As time goes on, the liquid component increases further as the process of liquefaction necrosis evolves. These collections, however, can differ substantially in the relative amount of liquid and solid components. For instance, in the first several days of the disease, peri-pancreatic ANCs may not be immediately evident. For instance, ANCs involving the pancreatic parenchyma are more obvious, because the pancreatic parenchyma fails to enhance, indicative of parenchymal necrosis. In contrast, the peri-pancreatic ANCs are not as obvious on CECT very early in the disease, because the peri-pancreatic tissue does not display obvious enhancement with the intravenous contrast agent even under normal circumstances. ANCs may be suspected (and assumed to be present), because they occur in the setting of severe acute pancreatitis [2] and especially when associated with pancreatic parenchymal necrosis (non-enhancement by IV contrast) or when there is what appears to be a thickened 'inflammatory' reaction extending down the left or right paracolic gutter on CT [4]. Therefore, the detection and differentiation of ANCs from APFCs can be difficult within the first few days of acute necrotizing pancreatitis;
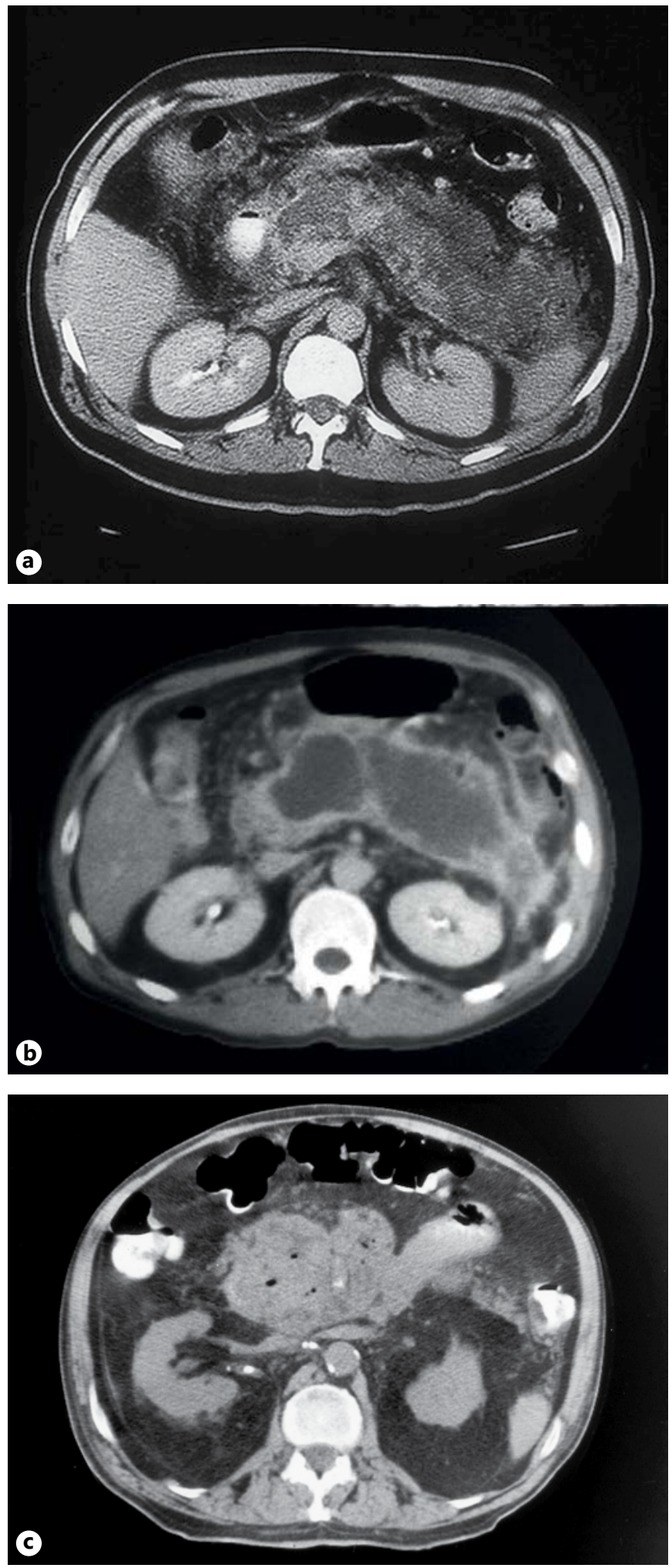

Fig. 3. ANC. a Sterile ANC. Note both pancreatic and peri-pancreatic non-homogenous collections in head and body/tail region. b WON. Six weeks later, encapsulation of collections. c Infected APN. Note ill-defined collection containing gas. 
however, several days later, ANCs become more obvious and especially so when suspected in the clinical course of the disease.

\section{Natural History}

ANCs remain sterile or become infected. The presence or absence of infection can be confirmed either with imaging studies (presence of gas within the collection is considered diagnostic) or the presence of a positive culture on microbiologic analysis of fine needle aspirations of the ANC, which is typically performed under imaging guidance (U/S or CT). As the ANCs mature and develop liquefaction necrosis, the body tends to surround these areas by forming a wall of reactive tissues and the ANC then becomes encapsulated. At that stage, the collection is termed WON. With time, the ANCs either resorb or develop into a WON.

\section{Walled-Off Necrosis}

\section{Etiopathogenesis}

ANCs that acquire a wall of reactive tissue are called WON. This term is self-explanatory and denotes the presence of necrosis with a reactive enhancing wall. This process usually takes 3-4 weeks and only arises in the setting of acute necrotizing pancreatitis. WON can involve areas of pancreatic parenchyma, peri-pancreatic tissues or, most commonly, both. Because peri-pancreatic necrosis can variably extend beyond the pancreas, WON can still be seen at sites distant from the pancreas.

\section{Imaging Characteristics}

Some combination of solid and fluid components is always present. What differentiates WON from a pancreatic pseudocyst is the presence of necrosis (solid elements) within the collection. As with a pancreatic pseudocyst, WON complicates pancreatic parenchymal necrosis usually or often has a connection with the pancreatic ductal system, but what differentiates WON from a pseudocyst is the presence of necrosis. Because imaging studies, especially CECT, may not always identify the solid component in these peri-pancreatic collections during the later phase of the disease, WON may be described erroneously as a pseudocyst. The objective presence of pancreatic necrosis (lack of enhancement by IV contrast), severe and protracted clinical course, or the later clinical deterioration despite optimal management usually suggest the presence of WON (possibly infected WON) rather than pseudocyst even when CECT findings are equivocal. In these circumstances, obtaining a U/S or MRI may be helpful in identifying the presence of solid components in the pancreatic/

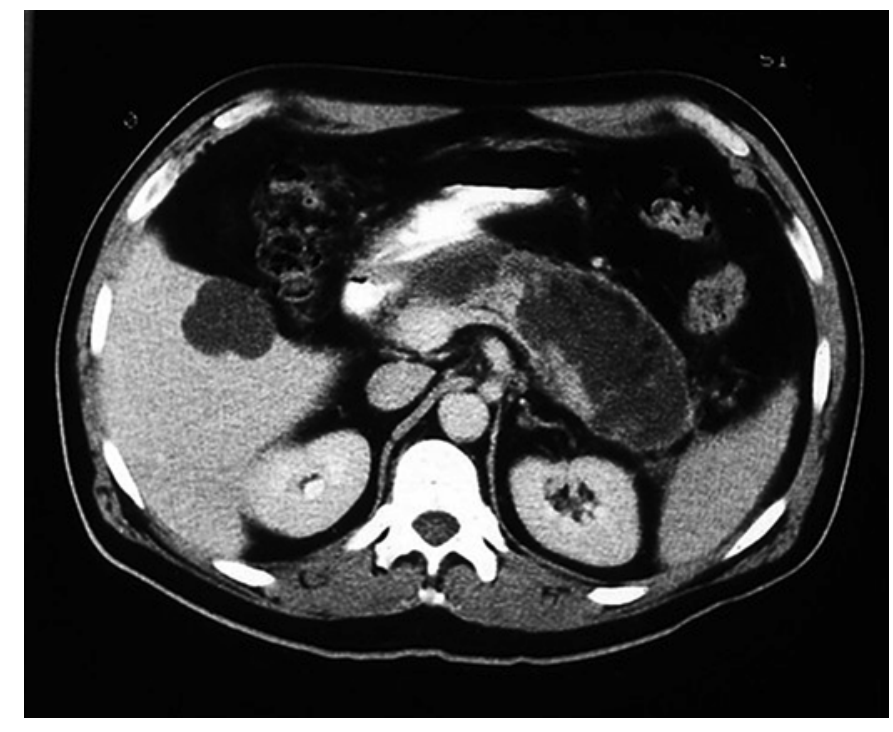

Fig. 4. WON. Note fluid and solid collections with thick walls involving the region of the body of the pancreas.

peri-pancreatic fluid collections. Distinctions between WON and a pancreatic pseudocyst are of crucial importance, because the management is completely different. For this reason, transabdominal or endoscopic U/S or MRI may be a useful adjunct during this window of the disease to identify the presence of necrosis.

Typically WON appears as heterogenous collections of both solid and liquid components that have a defined thickened, encapsulating wall (fig. 4, table 2). Unlike pseudocysts, which tend to be round and usually singular, WON is usually often non-round, can have several lobular components to each collection, can be multiple and are 'multiloculated' secondary to the associated solid necrotic material within the collection. These WON can be sterile or infected; the presence of gas within the collection is pathognomonic of infected WON (fig. 4).

\section{Natural History}

WON complicates acute necrotizing pancreatitis and not acute interstitial edematous pancreatitis. Because necrotizing pancreatitis is associated with greater morbidity and mortality, the presence of WON is a marker of overall potential disease severity. As with ANC, WON remains sterile or becomes infected.

About $20-30 \%$ of WON will become secondarily infected and usually requires some form of active, interventional necrosectomy - operative, endoscopic, laparoscopic or percutaneous. What is most important about WON is that its treatment is very different from that of a pancreatic 
pseudocyst in concept. Pancreatic pseudocysts that require treatment for pain or size-related symptoms can be managed by endoscopic (or operative) internal drainage alone. In contrast, WON requires a formal necrosectomy with removal/debridement of necrosis within the collections; simple internal drainage with, for instance, a transgastric, endoscopically-placed stent will likely convert a sterile WON into an infected WON. Therefore, transgastric endoscopic treatment of localized WON involves a much greater endoscopic access into the collection, which allows the necrotic tissue to evacuate into the stomach (or rarely the duodenum) and can be aided by 'driving' the endoscope into the collection combined with the use of mechanical means of endoscopic necrosectomy [14].

\section{Summary/Overview}

Thelast 2 decades of experience since the original Atlanta Classification in 1992 [1] have taught us as pancreatologists that many of our concepts of acute pancreatitis were naïve and even incorrect [2]. We now know that there are 2 basic forms of acute pancreatitis that denote different natural histories and prognoses - acute interstitial edematous pancreatitis and acute necrotizing pancreatitis. The pancreatic and peri-pancreatic collections that complicate acute pancre- atitis are usually not pancreatic pseudocysts - and this term should be reserved for the very special peri-pancreatic fluid collection with minimal or no necrosis that is a rare later ( $>4$ weeks) complication of acute interstitial edematous pancreatitis, which usually does not require active intervention. In contrast, the collections, both peri-pancreatic and pancreatic, that complicate acute necrotizing pancreatitis, ANC and WON, are not pseudocysts and may very well (20-30\%) require active intervention by some form of active necrosectomy. Recent work has shown that some respond to antibiotic treatment alone, while most require either percutaneous, endoscopic, minimal access 'step up' treatment [15] or formal operative necrosectomy [16-18]. The old non-specific term of 'phlegmon' is no longer appropriate, and we now understand that patients in the past who had a 'phlegmon' probably had a peri-pancreatic ANC. The treatment of pancreatic and peri-pancreatic collections are different depending on the setting (acute interstitial edematous pancreatitis vs. acute necrotizing pancreatitis). These terms APFC, pancreatic pseudocyst, ANC and WON should be used carefully and specifically.

\section{Disclosure Statement}

None.

\section{References}

$>1$ Bradley EL 3rd: A clinically based classification system for acute pancreatitis. Summary of the international symposium on acute pancreatitis, Atlanta, Ga, September 11 through 13, 1992. Arch Surg 1993;128:586-590.

-2 Sarr MG, Banks PA, Bollen TL, Dervenis C, Gooszen HG, Johnson CD, et al: The new revised classification of acute pancreatitis 2012 . Surg Clin North Am 2013;93:549-562.

-3 Sakorafas GH, Tsiotos GG, Sarr MG: Extrapancreatic necrotizing pancreatitis with viable pancreas: a previously under-appreciated entity. J Am Coll Surg 1999;188:643-648.

$\checkmark 4$ van Santvoort HC, Bollen TL, Besselink MG, Banks PA, et al: Describing peripancreatic collections in severe acute pancreatitis using morphologic terms: an international interobserver agreement study. Pancreatology 2008; 8:593-599.

5 Bakker OJ, van Santvoort H, Besselink MG, et al: Extrapancreatic necrosis without pancreatic parenchymal necrosis: a separate entity in necrotising pancreatitis. Gut 2013;62:14751480.

6 Bollen TL, van Santvoort HC, Besselink MG, et al: The Atlanta classification of acute pancreatitis revisited. Br J Surg 2008;95:6-21.
7 Lenhart DK, Balthazar EJ: MDCT of acute mild (nonnecrotizing) pancreatitis: abdominal complications and fate of fluid collections. AJR Am J Roentgenol 2008;190:643-649.

$>8$ Pelaez-Luna M, Vege SS, Petersen BT, Chari ST, Clain JE, Levy MJ, et al: Disconnected pancreatic duct syndrome in severe acute pancreatitis: clinical and imaging characteristics and outcomes in a cohort of 31 cases. Gastrointest Endosc 2008;68:91-97.

$>9$ Banks PA, Bollen TL, Dervenis C, Gooszen HG, Johnson CD, Sarr MG, et al: Classification of acute pancreatitis - 2012: revision of the Atlanta classification and definitions by international consensus. Gut 2013;62:102111.

10 Bradley EL, Clements JL Jr, Gonzalez AC: The natural history of pancreatic pseudocysts: a unified concept of management. Am J Surg 1979;137:135-141.

11 Vitas GJ, Sarr MG: Selected management of pancreatic pseudocysts: operative versus expectant management. Surgery 1992;111:123130.

$>12$ Yeo CJ, Bastidas JA, Lynch-Nyhan A, Fishman EK, Zinner MJ, Cameron JL: The natural history of pancreatic pseudocysts document- ed by computed tomography. Surg Gynecol Obstet 1990;170:411-417.

13 Baron TH, Morgan DE: Acute necrotizing pancreatitis. N Engl J Med 1999;340:14121417.

14 Papachristou GI, Takahashi N, Chahal P, Sarr MG, Baron TH: Peroral endoscopic drainage/ debridement of walled-off pancreatic necrosis. Ann Surg 2007;245:943-951.

15 van Santvoort HC, Besselink MG, Bakker OJ, et al: A step-up approach or open necrosectomy for necrotizing pancreatitis. $\mathrm{N}$ Engl J Med 2010;362:1491-1502.

16 Rau B, Bothe A, Beger HG: Surgical treatment of necrotizing pancreatitis by necrosectomy and closed lavage: changing patient characteristics and outcome in a 19-year, single-center series. Surgery 2005;138:28-39.

17 Tsiotos GG, Luque-de Leon E, Soreide JA, et al: Management of necrotizing pancreatitis by repeated operative necrosectomy using a zipper technique. Am J Surg 1998;175:9198.

-18 Fernandez-del Castillo C, Rattner DW, MaKary MA, et al: Debridement and closed packing for the treatment of necrotizing pancreatitis. Ann Surg 1998;228:676-684. 